\title{
Thermo-optical Phase Shifter with Integrated Diodes for Multiplexed Control
}

\author{
Antonio Ribeiro and Wim Bogaerts \\ Ghent University - IMEC, Photonics Research Group, Department of Information Technology, Ghent, Belgium \\ Center for Nano and Biophotonics (NB-Photonics), Ghent, Belgium \\ Antonio.Ribeiro@UGent.be
}

\begin{abstract}
We present a thermo-optic silicon phase shifter with integrated diode, which allows multiplexed access to heaters and reduces the number of pads to drive complex silicon photonics circuits. We also demonstrate that such heaters can be driven using digitally modulated signals, which increases the linearity of the phase shift response of the device.
\end{abstract}

OCIS codes: (130.3120) Integrated optics devices, (130.4815) Optical switching devices, (130.4110) Modulators.

\section{Introduction and Concept}

Heaters are an attractive solution for phase shifting in silicon photonics due their simplicity of implementation and their compactness, compared to alternatives such as free-carrier modulators [1-3]. Although compact, heaters require bulky pads to connect them to external driver electronics. One way to save space is multiplexing the use of the pads, driving multiple heaters with the same set of pads.

A typical side heater is obtained by placing a doped silicon strip close to the target waveguide, and using it as an electric resistor. The resistance of the heater is determined by its electrical properties (both inherent from the silicon and obtained from its dopants) and its geometry. Using both $P$-type and $N$-type dopants to create a $P N$ junction creates a heater that is, at the same time, a diode (Fig.1).

\section{Design and Fabrication}

The heater consist of a silicon strip doped with $N$-type dopants, while one of its edges was doped with $P$-type, to create the $P N$ junction (Fig.1). We implemented the device in imec's ISIPP25G+ silicon photonics platform. The heater was placed $0.7 \mathrm{um}$ away from the target waveguide. The implemented heaters are 50um long and $1 \mathrm{um}$ wide, fabricated on a $220 \mathrm{~nm}$ thick silicon layer. The I-V response of the heater is shown in Fig.1(b). The breakthrough voltage stayed at $-7 \mathrm{~V}$ and it yielded $9.6 \mathrm{~mW}$ when forward-biased at $7 \mathrm{~V}$. To achieve higher power output without compromising the the operational range of $\pm 7 \mathrm{~V}$ we placed multiple diodes in parallel. For the fabricated phase shifter we report a $\pi \mathrm{rad}$ phase shift at $20.9 \mathrm{~mW}$, which is a result compatible with the reported values for this technology [4].
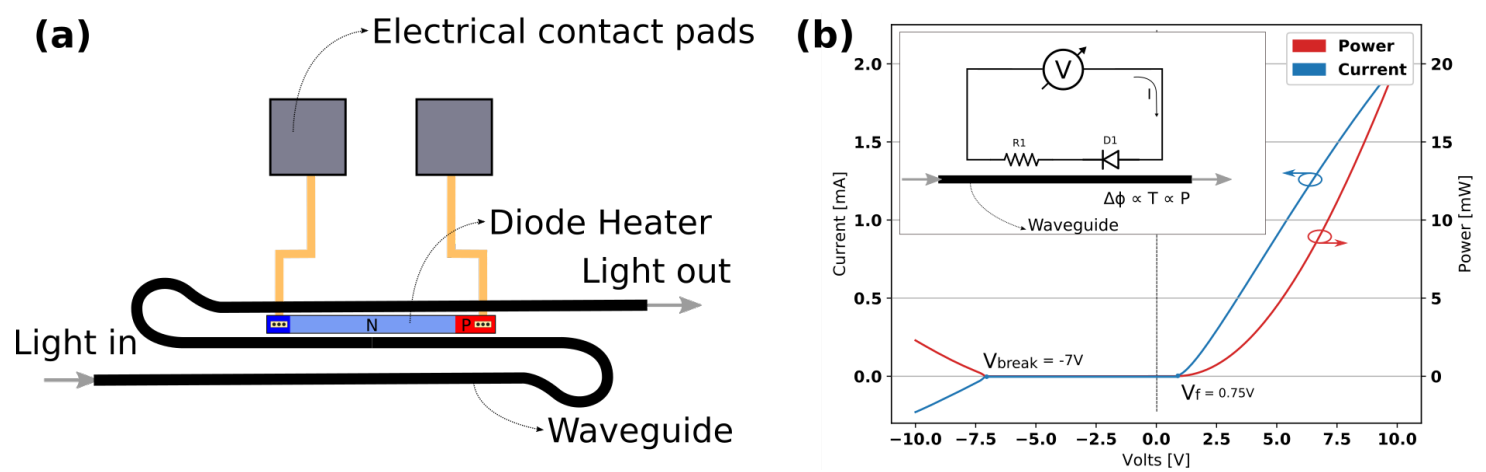

Fig. 1: The conceptual design (a) and an IV curve (b) of the diode heater. 

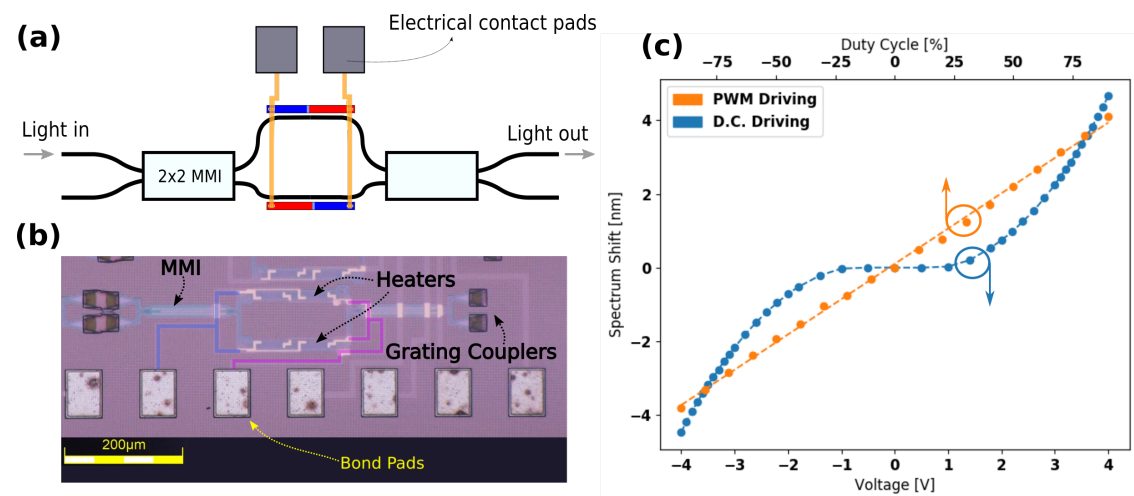

Fig. 2: MZI with diode heaters in each arm. The conceptual design (a) and the fabricated chip (b). The curve (c) shows the spectrum shift in function of a PWM and DC driving signal.

\section{Applications and Obtained Results}

As a demonstration. we placed the diode heaters in two circuits: a pair of ring resonators with distinct resonances (Fig.3) and a Mach-Zehnder Interferometer (MZI) operating in push-pull (Fig.2) .

\subsection{Red-Blue shifting of MZIs filters}

In the MZI circuit (Fig.2) the heaters are placed on both arms of the interferometer, allowing it to operate in push-pull. By routing the electrical connection of the two heaters in parallel, but with opposite polarity, we can use one pair of bond pads to drive either the top heater or the bottom one, depending on the polarity of the driving signal. It allows to either red-shift or blue-shift the spectrum response of the device with only one pair of contact pads.

We operated the circuit driving the pair of heater with a variable voltage source (from $-4 V$ to $+4 V$ ) and also with a pulse width modulated (PWM) signal (fixed voltage, variable duty cycle. A negative duty cycle indicates a signal with negative polarity). From that we recorded the induced shift in the spectrum of the MZI filter, shown in Fig.2(c). It is clear from the plot that changing the bias will result in either a positive (red) or a negative (blue) shift. Also, it is noticeable that the response of the circuit to a digital PWM driving signal is much more linear than for DC driving. This is true because the induced phase shift is proportional to the temperature, which is proportional to the dissipated power. For a DC driving scheme, the power is quadratic to the applied voltage, while in the PWM scheme, the power grows linearly with the duty cycle. PWM driving is possible due to the high time constant of the heater (77.5us, measured). When the digital driving signal changes much faster than the thermal response time, the induced phase shift is averaged over time. For driving our circuit we used a PWM signal with fixed amplitude of $+5 \mathrm{~V}$ (or $-5 \mathrm{~V}$ for negative bias) and frequency $f=2 M H z$.

\subsection{Simultaneous control using PWM signal with two polarity}

In Fig.3(b) we show the spectrum of the two rings responding independently, based on the bias of the driving signal. This technique allows to address either one heater or the other, but if we time-multiplex the driving signal we can address both heaters at the same time. To achieve that we used a duobinary PWM signal that has both a positive and a negative cycle that that can be independently defined (Fig.3(c), bottom), allowing us to address, independently, the two heaters. Fig.3(c) shows the correlation between the duty cycle values (rows 1 and 3) and the measured phase shift (rows 2 and 4), as well as the PWM signal (bottom). This demonstrates that we can use one pair of pads to control two heaters at the same time, in an independent fashion.

\section{Extending the Concept for Matrix Addressing}

The multiplexing can be extended by placing the phase shifters in a matrix topology, connecting the heaters as $M$ rows and $N$ columns arrangement (Fig.4). We can now use the asymmetric behaviour of the diode to address one row at time and individually drive each heater in that row. If we time-multiplex the addressing of the rows, and rely on the high time constant of the heaters to delivery a steady phase shift, we can sequentially address all the heaters of the circuit. This way we reduce the number of pads from $M x N+1$ (ground) to $M+N$. 

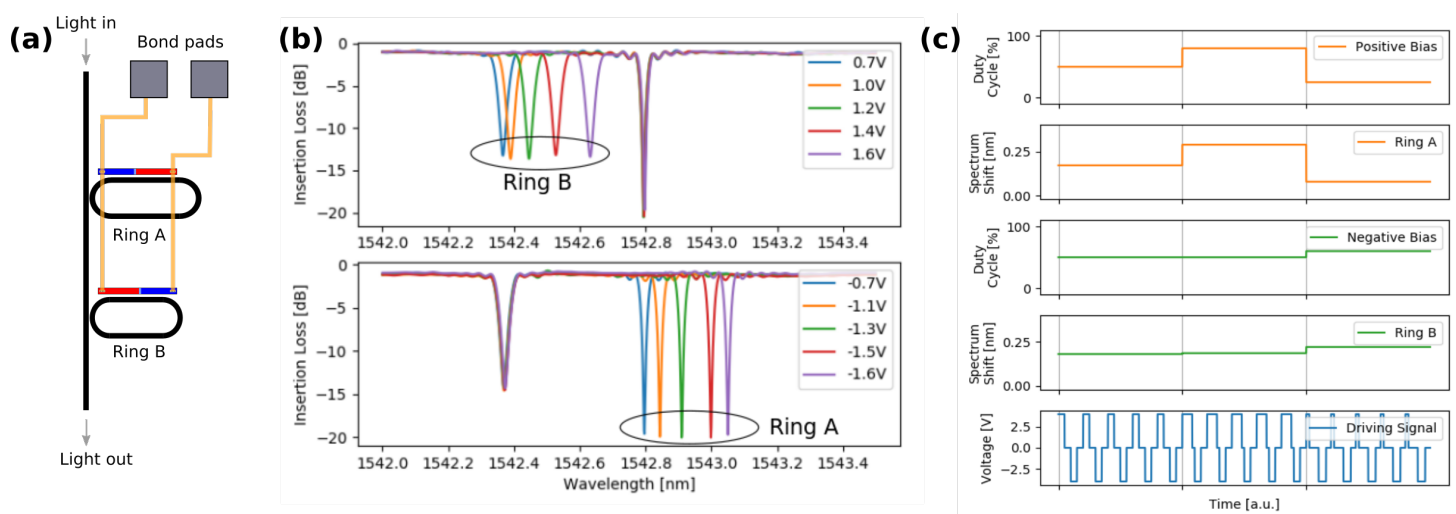

Fig. 3: The circuit with two rings (a), the spectrum shift of both rings for different DC driving voltages (b). The plot (c) shows the correspondence between the duty-cycle values of the duobinary PWM signal (rows 1 and 3), measured spectrum shift for rings (rows 2 and 4) and the respective duobinary PWM signal (row 5).
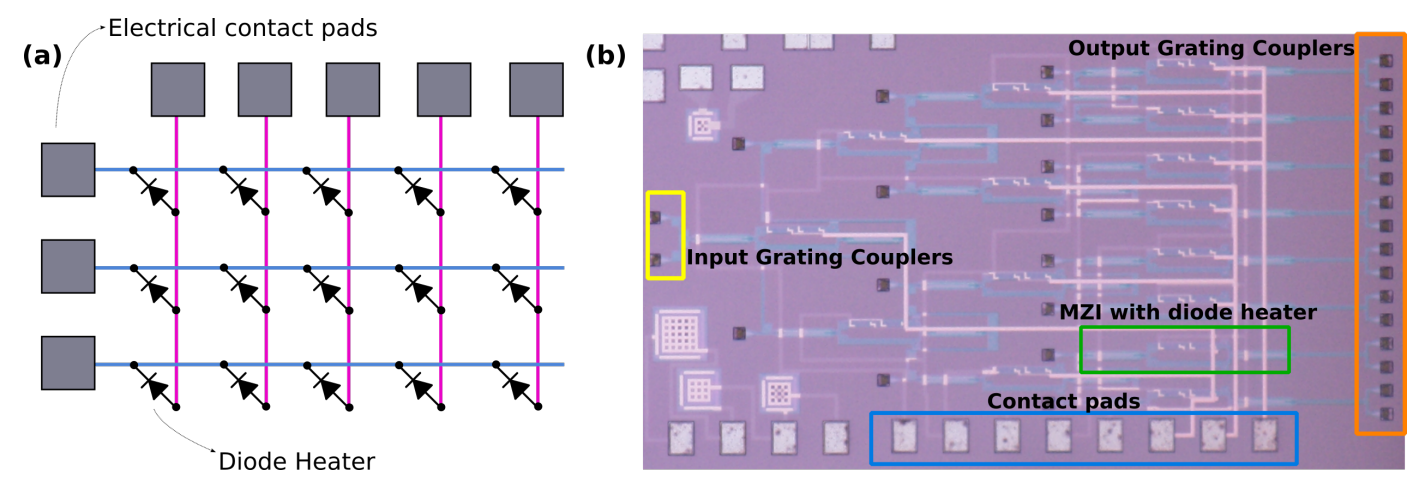

Fig. 4: The heaters can be disposed in a $M \times N$ arrangement for the multiplexing driving (a). Microscope image of a fabricated demonstration switch with 15 MZIs with diode heaters, placed in a $3 \times 5$ matrix configuration (b).

Our experimental results indicate that we are able to deliver $\pi$ rad phase shift using a PWM signal with amplitude of $7 \mathrm{~V}$ and a duty cycle of $28 \%$, so with these heaters we can multiplex up to 3 rows of $N$ heaters using this matrix addressing technique.

\section{Conclusion}

We demonstrated the use of silicon photonics heaters with integrated diodes to allow bias-dependent operation. That, combined with digital driving, can provide multiplexed access to the heaters, reducing the number of necessary pads. We also demonstrated that PWM driving improves the linearity response of the heaters.

\section{References}

1. M. R. Watts, J. Sun, C. DeRose, D. C. Trotter, R. W. Young, and G. N. Nielson, ”Adiabatic thermo-optic MachZehnder switch", Opt. Lett. 38, 733-735 (2013)

2. Y. Maegami, G. Cong, M. Ohno, M. Okano, and K. Yamada, "Strip-loaded waveguide-based optical phase shifter for high-efficiency silicon optical modulators," Photon. Res. 4, 222-226 (2016)

3. Y. Yang, Q. Fang, M. Yu, X. Tu, R. Rusli, and G. Lo, "High-efficiency Si optical modulator using Cu travellingwave electrode," Opt. Express 22, 29978-29985 (2014).

4. A. Masood, M. Pantouvaki, G. Lepage, P. Verheyen, J. Van Campenhout, P. Absil, D. Van Thourhout, W. Bogaerts, "Comparison of heater architectures for thermal control of silicon photonic circuits", Group IV Photonics, South Korea, p.ThC2 (2013) 